\title{
Le baiser de Judas et l'ici/là de la chair
}

Un dévoilement esthétique de l'espacement phénoménologique

Benjamin Delmotte

\section{(2) OpenEdition}

Journals

Édition électronique

URL : http://journals.openedition.org/alter/463

DOI : $10.4000 /$ alter.463

ISSN : 2558-7927

Éditeur :

Association ALTER, Archives Husserl (CNRS-UMR 8547)

\section{Édition imprimée}

Date de publication : 3 décembre 2017

Pagination : 235-247

ISBN : 978-2-9550449-3-3

ISSN : 1249-8947

Référence électronique

Benjamin Delmotte, « Le baiser de Judas et l'ici/là de la chair », Alter [En ligne], 25 | 2017, mis en ligne le 01 décembre 2018, consulté le 20 avril 2019. URL : http://journals.openedition.org/alter/463 ; DOI :

10.4000/alter.463 


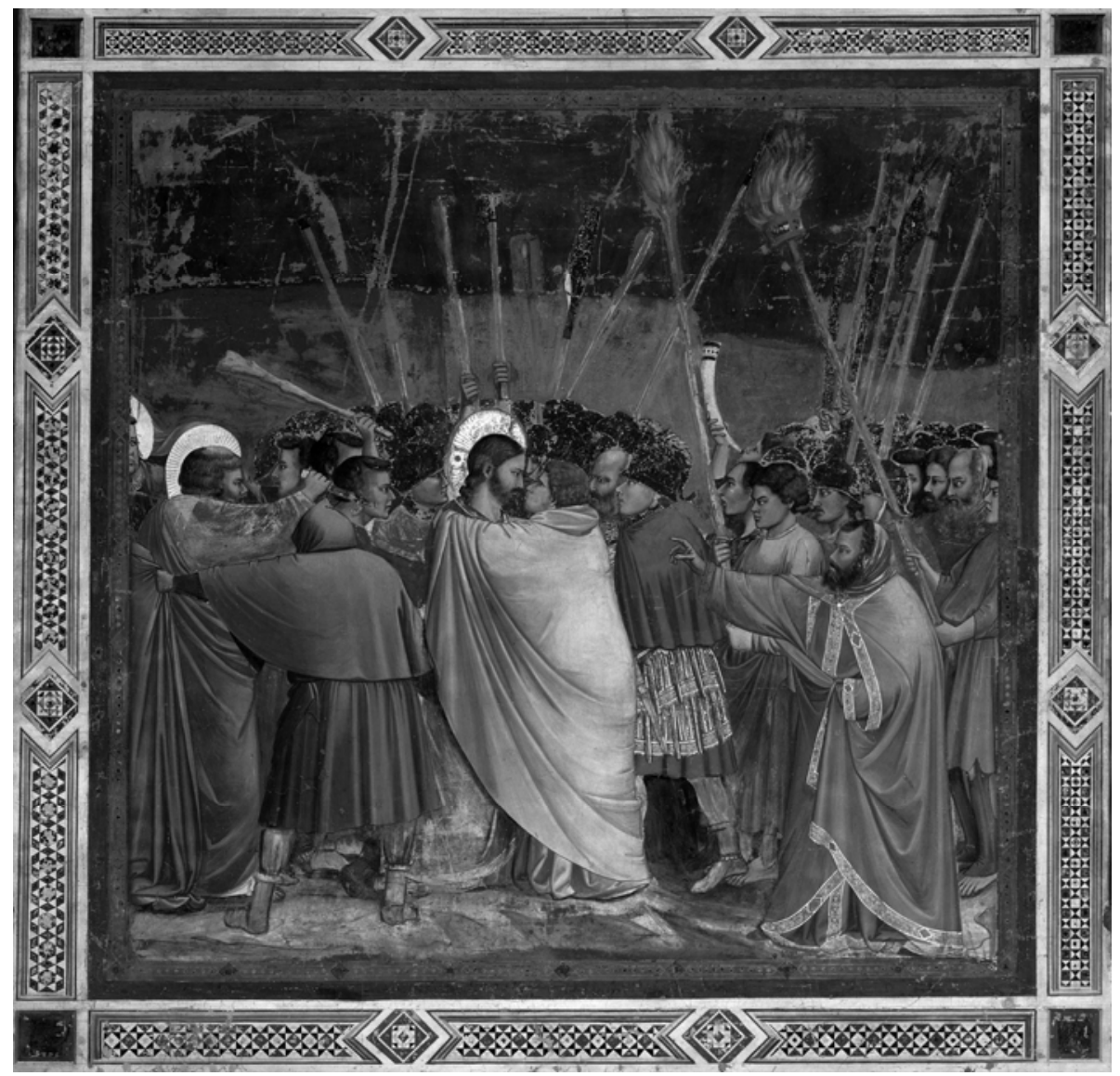

Giotto, Le Baiser de Judas, 1303-1306, fresque, chapelle des Scrovegni, Padoue. 


\section{LE BAISER DE JUDAS ET L'ICI/LÀ DE LA CHAIR UN DÉVOILEMENT ESTHÉTIQUE DE L'ESPACEMENT PHÉNOMENOLOGIQUE}

Benjamin Delmotte

\section{Introduction : \\ Vers une compréhension plastique de l'espace phénoménologique}

On doit à Husserl une compréhension de l'espace essentiellement caractérisée par la centralité de la chair, qu'il définit notamment comme «centre d'orientation $»^{1}$ (Der Leib als Orientierungszentrum). Cette centralité offre à la chair un caractère à la fois principiel et absolu : principiel d'abord, tant la chair détermine les régions dans l'espace («Tout être spatial apparaît nécessairement de telle manière qu'il apparaît près ou loin, en haut ou en bas, à droite ou à gauche »)2. Absolu ensuite, tant l'ici qu'elle constitue se vit par-delà la relativité des positions corporelles effectives.

Mais comment comprendre cette absoluité de la chair ? Deux difficultés, souvent commentées, semblent en effet de nature à la remettre en question. La première tient à l'ancrage physique de la chair et à l'ambiguïté de sa distinction d'avec le corps. Pour Husserl, cette inscription de l'ici absolu de la chair dans un corps effectif aux positions variables et contingentes ne signifie toutefois en rien sa relativisation.

\footnotetext{
${ }^{1} \mathrm{E}$. Husserl, Idées directrices pour une phénoménologie et une philosophie phénoménologique pures, Livre second, Recherches phénoménologiques pour la constitution, traduction Éliane Escoubas, Paris, PUF, «Épiméthée », 1982, p. 223.

2 Idem.
} 
L'ici du corps propre est absolu car, en tant que centre d'orientation, il se concentre en un pur point distinct de toute localisation effective :

En ce qui concerne l'éloignement, il s'agit ici, objectivement, de l'êtreplus-loin ou être-plus-pres d'un objet qui acquiert son second pointde-relation en moi [...]. Plus précisément, ce point-de-relation n'est pas le corps tout entier, il est placé dans la partie de celui-ci qui n'est pas vue, il se trouve quelque part dans la tête, dans l'œil ou derrière l'œil ${ }^{3}$.

On pourrait dire que le centre échappe à la localisation réelle tant il demeure flottant, à la fois partout et nulle part. De fait, il peut coïncider avec l'ici ou le là de telle ou telle partie du corps : dans la tête, ou dans l'œil, ou derrière l'œil, il peut encore résider dans le regard (en tant qu'il se distingue de l'œil), sinon dans un tout autre point du corps. Ainsi celui qui, par exemple, a le pied cassé va-t-il être amené à re-déterminer son expérience spatiale de manière à protéger son membre blessé. Marcher dans le métro signifiera envisager la proximité des autres usagers à partir de ce pied, de manière à éviter qu'il ne soit heurté. De la même manière que la femme enceinte recentre son expérience spatiale afin de protéger son ventre. Aussi absolu soit-il, l'ici charnel connaît donc, chez un même individu, une variation notable de ses manifestations effectives.

La seconde difficulté tient à la présence d'autrui comme centre d'orientation lui-même absolu. Mais là encore, pour Husserl, cette présence d'autrui ne remet pas davantage en question l'absoluité de la centralité. L'autre qui, pour moi, se donne comme là-bas (illic) vit son corps comme une donnée "originelle »", "dans le mode d'un "hic absolu" $»^{5}$. Il faut donc comprendre la centralité de la chair à la fois comme impartageable et distribuée : l'espace s'organise dans une discontinuité de sphères propres qui se recouvrent sans toutefois s'identifier absolument.

Néanmoins, cette caractérisation du vécu de l'expérience spatiale ne laisse pas de poser problème : comment comprendre la relation entre le «hic absolu» et l'ici effectif du corps? Et comment comprendre que le hic absolu de la chair puisse envisager un autre ici dans l'illic de l'autre ? Comment, enfin, comprendre que les sphères propres, chacune absolument centrée, puissent coexister et même se rencontrer? À l'affirmation d'un espace essentiellement centré sur un ici, il

\footnotetext{
${ }^{3}$ E. Husserl, Chose et espace, Leçons de 1907, traduction Jean-François Lavigne, Paris, PUF, "Épiméthée », 1989, p. 271.

${ }^{4}$ E. Husserl, Méditations cartésiennes, traduction Gabrielle Pfeiffer et Emmanuel Levinas, Paris, Vrin, 1992, p. 191.

${ }^{5}$ Idem.
} 
semble donc nécessaire d'articuler une description phénoménologique de l'espacement qui va d'ici à là : de la chair au corps, mais aussi du hic à l'illic de l'autre.

Que signifie alors un tel espacement? Comment mener sa description? Et quelle peut être sa portée phénoménologique? Indiquons-le d'emblée : cet espacement n'est en rien une relativisation de l'absoluité de la chair, mais doit au contraire nous amener à réaffirmer sa puissance, en soulignant sa nature dynamique et plastique. Dans cette perspective, la réflexion qui suit entend mettre en évidence l'intérêt et la pertinence de l'analyse esthétique dans la révélation phénoménologique de cet espacement. Car l'œuvre d'art, tant pour ses ambiguïtés spatiales que sa capacité à suggérer l'empathie, est sans doute remarquablement à même de nous rendre sensible à cet espacement. C'est pourquoi nous avons choisi de conduire notre description à partir d'une même œuvre, à savoir une fresque peinte par Giotto dans la chapelle des Scrovegni, à Padoue, au tout début du XIVe siècle.

\section{L'espacement et sa problématisation esthétique}

À un premier niveau, les ambiguïtés spatiales de l'expérience esthétique touchent d'abord à la détermination du là que constitue l'œuvre, en tant qu'objet plus ou moins éloigné de l'ici que nous définissons comme spectateur. Ainsi, pour reprendre la terminologie mise en place par Husserl (lorsqu'il décrit sa contemplation d'une reproduction de la Théologie de Raphaël) ${ }^{6}$, il nous faut reconnaître que la spatialité de $l^{\prime}$ «image physique » ne recouvre pas celle de l'« objetimage » qui figure le sujet.

Si Husserl insiste sur la modification de visée qu'implique le passage de l'image physique à l'objet-image, il ne faut pas oublier que cette modification est le plus souvent irréfléchie et inaperçue. Dans la contemplation esthétique, nous ne ressentons pas vraiment cette bascule de l'espace réel vers l'espace pictural. La modification intentionnelle est effectuée presque sans solution de continuité apparente, et la discontinuité «modale» de l'espace, plus que dans une conscience actuelle de modification intentionnelle, apparaît plutôt de façon rétroactive, dans la surprise d'un vertige, lorsque, pour une raison ou pour une autre, nous quittons l'espace figuré et sommes ramenés à la spatialité réelle de notre corps face à l'œuvre d'art.

\footnotetext{
${ }^{6}$ Nous renvoyons ici aux analyses conduites par Husserl in Phantasia, conscience d'image, souvenir, De la phénoménologie des présentifications intuitives, Textes posthumes (1898-1925), traduction Raymond Kassis et Jean-François Pestureau, Grenoble, Jérôme Millon, 2002, p. 83-84.
} 
Il y a surprise et vertige car la discontinuité nous apparaît alors subitement, quand la contemplation nous avait fait glisser quasi imperceptiblement d'un espace à l'autre. On pourrait dire que nous étions si intimement plongés dans l'espace de l'objet-image que nous en avions oublié son caractère d'objet, la distance et la relation de visà-vis qu'il implique, en cédant à ce que l'on pourrait appeler « l'aspiration de l'image ».

C'est pourquoi cette ambiguïté du là que constitue l'œuvre n'est pas sans conséquence sur notre propre $i c i$ : dans la contemplation esthétique, tout se passe comme si l'ici était - en partie - aspiré par le là de l'œuvre. Comment comprendre cette « relativisation » de l'absolu?

Pour répondre à cette question, sans doute est-il opportun de revenir sur les analyses conduites par Merleau-Ponty lorsqu'il constate l'ambiguïé locale du spectateur esthétique :

je serais bien en peine de dire où est le tableau que je regarde. Car je ne le regarde pas comme on regarde une chose, je ne le fixe pas en son lieu, mon regard erre en lui comme dans les nimbes de l'Etre, je vois selon ou avec lui plutôt que je ne le vois ${ }^{7}$.

Le «où» du tableau, sa localisation effective, disparaît dans la mesure où disparaît aussi bien celle du spectateur : nous assistons à un éclatement de l'espace de l'objectivité, en tant que ce dernier se définit par la distance du sujet à l'objet et l'espacement correspondant de l'ici et du là. On relève en effet une quasi disparition du repère physique que constitue, $i c i$, le sujet, dans la mesure où ce dernier peut ressentir sa spatialité physique effective se résorber en simple tension visuelle dont le principe d'animation semble revenir au tableau lui-même dans sa capacité à aspirer le regard. Autrement dit, de même que, là-bas, l'image physique du tableau s'oublie au profit de l'objet-image qui figure, on peut remarquer, ici, la perte de détermination physique de la subjectivité dans sa tension/aspiration vers/par l'image.

Que conclure de cette apparente relativisation de l'ici dans la contemplation esthétique ? Faut-il dire que l'ici et le là se rejoignent dans un même processus de "désobjectivation »? Qu'une même spatialité fondamentale serait atteinte, par-delà l'espacement de l'ici et du là ?

Sans doute notre référence à Merleau-Ponty pourrait-elle nous amener à voir dans cette expérience une sorte d'accès à quelque entrelacs de l'ici et du là : pour le peintre qui s'adonne à son art, «la même chose est là-bas au cœur du monde et ici au cœur de la vision $»^{8}$. Mais

\footnotetext{
${ }^{7}$ M. Merleau-Ponty, L'œil et l'esprit, Paris, Gallimard, «Folio essais », 1993, p. 23.

8 Ibid., p. 28.
} 
quoiqu'il en soit de la position de Merleau-Ponty (sans doute plus nuancée, au final, que ce que nous suggérons ici), il nous semble nécessaire de rejeter l'assimilation de l'ici et de là et d'insister plutôt sur la permanence de leur espacement.

Soit donc cette fresque peinte par Giotto et représentant le Baiser de Judas dans la chapelle des Scrovegni. Si mon regard se concentre sur la représentation de cet épisode biblique, la chapelle réelle, sa localisation dans la ville de Padoue, son architecture intérieure, mais aussi l'espace pictural constitué par les fresques voisines passent tout à fait à l'arrière-plan de mon attention tant celle-ci glisse dans un espace autre, au point de $\mathrm{m}^{\prime}$ installer quasiment dans l'espace de la scène. Je ne suis plus tout à fait $i c i$, planté sur mes deux jambes, face à la paroi latérale d'une chapelle, mais aussi dans le Jardin des Oliviers, dans une étrange proximité avec les personnages bibliques représentés. Pour autant, je ne puis pas plus être totalement là-bas, tant la distance de la contemplation peut se rappeler à moi et l'évidence de l’hétérogénéité des espaces demeurer présente.

Telle est l'ambiguiité de cet éclatement de l'espace : le dépassement de l'opposition objective de l'ici et du là n'est pas pour autant leur confusion. D'une part parce que l'opposition entre espace réel et imaginaire demeure (elle se rappelle d'ailleurs de façon évidente pendant l'expérience de contemplation); et, d'autre part, parce que la conscience d'une séparation insiste jusque dans la dynamique de compréhension à l'œuvre dans la contemplation. J'ai beau ressentir une proximité étonnante avec ces personnages, et même avoir l'impression que mon regard est happé par la fresque, cette proximité et cette attirance ne sont pas fusion. L'évidence de la compréhension de la fresque rencontre celle de son détachement: en même temps qu'elle se donne à moi, elle m'échappe, m'oblige à revenir cent fois sur les mêmes détails, à alterner vision d'ensemble et vision des détails.

Soit, notamment, cette construction rayonnante, centrée sur l'échange de regards entre le Christ et l'apôtre qui vient de trahir. Il est, de fait, bien difficile de localiser exactement le centre de cette construction : dans l'auréole christique? Dans l'œil du Christ? Ou, quelque part, dans l'échange de regard entre le Christ et l'apôtre qui trahit? En outre, quoi qu'il en soit du centre exact de cette construction circulaire, elle rayonne et se diffuse dans tout le tableau (organisation circulaire des lances, piques et flambeaux, groupement des personnages...) sans suffire à justifier tous les détails, comme par exemple l'expression propre à tel ou tel témoin de la scène. En même temps donc que je saisis immédiatement un principe d'organisation formel du tableau, j'en vois les limites et admets que mon appréhension est 
condamnée à une forme de discontinuité indépassable. Surtout, en même temps que je pénètre intimement le là de l'œuvre, comme si j'en faisais partie, je suis ramené $i c i$, à une forme d'incompréhension et de relance de l'appréhension.

\section{L'espacement et sa problématisation empathique}

Ce que nous venons de décrire à propos de cette fresque pourrait sans doute valoir de façon générale comme caractéristique de la contemplation esthétique. Mais l'exemple choisi, dans sa particularité, permet sans doute de creuser davantage encore les ambiguïtés de l'espace phénoménologique, dans la mesure où l'ambiguïté esthétique de l'espace se conjugue à celle en jeu dans l'empathie.

L'espace perd en effet d'autant plus de détermination locale que l'opposition de l'ici et du là peut aussi recouvrir celle de deux principes d'organisation de l'espace. La fresque me permet de me « quasi » projeter d'ici à là au sens où il me semble pouvoir, comme de l'intérieur, ressentir notamment ce qui est ressenti par le Christ luimême. Je ne suis pas simplement ce corps réel et contemplatif, situé dans l'espace de la chapelle, mais aussi ce corps biblique représenté, qui, en même temps qu'il se tient fermement au milieu de cet espace, plante ses yeux dans ceux du traître et lui rappelle ainsi la distance qui les sépare, malgré leur proximité.

De fait, les deux corps sont très proches l'un de l'autre, dans le baiser qui vient d'avoir lieu. Ils semblent même ne faire qu'un, $d^{\prime}$ ailleurs recouvert d'un même manteau. Mais en même temps que nous pouvons voir, extérieurement, cette proximité effective, nous ressentons, intérieurement, l'éloignement. L'empathie nous fait ressentir le corps christique comme un repère inamovible, un ancrage assuré, qui voit son ancien disciple comme réduit par son geste de traîtrise à une forme d'éloignement. Dans l'empathie pour le Christ, notre regard est dur, implacable, non pas tant parce qu'il est haineux ou accusateur que parce qu'il se tient du côté de la vérité et de la justice. À travers la fermeté de notre position, nous pouvons sentir Judas glisser, être emporté loin de nous dans une dynamique de retrait. C'est pourquoi sa main qui nous ceint encore est pourtant déjà très loin, repoussée dans un espace autre, celui de la culpabilité, comme si le poids du remords l'avait déjà attrapée. Étrangement donc, alors que le Christ va être arrêté et emmené par les soldats, c'est Judas qu'on voit emporté, perdu, tandis que nous restons inamovibles. Nous constituons un centre juste et vrai dont le mal ne peut connaître l'heureuse proximité. 
Faut-il dire que le regard (plus que l'œil) christique constitue le centre absolu de cette fresque ? Sans doute. Mais il nous faut toutefois ajouter que cette projection empathique n'est pas la seule autorisée par la contemplation. Car si nous pouvons avoir l'impression de ressentir intérieurement ce que vit le Christ, nous pouvons tout autant, semble-t-il, vivre intérieurement la traîtrise de Judas. La bassesse de son geste ne se comprend pas seulement extérieurement, dans la symbolique de la couleur de son manteau, la courbure de son corps ou la différence de taille des deux personnages représentés de profil, mais également de l'intérieur, dans cette dynamique d'éloignement et de rabaissement produite par l'échange des regards. Nous pouvons également nous mettre à la place du traître, et nous voir rabaissés avec lui. Comme lui, nous pouvons sentir un gouffre s'installer entre nous et le Christ, et ce, malgré cette proximité réelle qui semble nous permettre de le toucher encore. Le visage du Christ est l'écran où nous lisons notre propre bassesse, avec d'autant plus de certitude qu'il reste silencieux et tranquille. Pour un peu, l'épaule que nous touchons encore nous brûlerait les doigts: notre main glisse dorénavant sur elle comme sur un objet impénétrable, déjà lointain. Autrement dit, cette épaule semble maintenir une distance absolue dans son contact même. Nous nous sentons alors pris dans un mouvement qui nous exclut de la grandeur incarnée par celui qui nous fait face, qui nous périphérise, ou mieux, nous éjecte.

Ajoutons enfin que cette double projection empathique peut être compliquée par ceci que nous pouvons quasi-disparaitre - nous retirer - en tant que principe empathique et avoir l'impression que le Christ est à même de se projeter de façon empathique en Judas, comme ce dernier est à même de se projeter dans celui qu'il vient de trahir. À travers la contemplation extérieure de cet échange de regards, il nous semble être le simple témoin, et de la façon dont le Christ saisit l'éloignement de son apôtre après le choix du mal, et de la façon dont Judas constate qu'il ne peut plus demeurer dans la proximité et la hauteur du Christ.

En quoi cette dimension empathique de la contemplation esthétique - avec toutes ses ambivalences - renforce-t-elle l'ambiguïté de

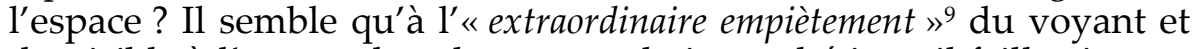
du visible à l'œuvre dans la contemplation esthétique il faille ajouter, sinon opposer, l'ambiguïté amenée par le jeu de décentrement/recentrement à l'œuvre dans l'empathie qui accompagne et renforce cette contemplation esthétique. Il nous semble en effet que l'espace phéno-

${ }^{9}$ M. Merleau-Ponty, L'eil et l'esprit, op. cit., p. 17. 
ménologique n'est pas tant celui que nous découvririons dans une «proximité heureuse » avec le monde que celui qui se fait jour dans un processus de recentrement lié à la reconfiguration de la chair.

Découvrir la fresque de Giotto ce n'est pas seulement défaire la distance objective ; ce n'est pas non plus - et ça n'est sans doute pas $\mathrm{du}$ tout - rendre indistinct l'ici et le là. C'est davantage réaffirmer (paradoxalement) l'absoluité de l'ici dans sa capacité à se (quasi) découvrir jusque dans ce qui résiste à toute assimilation achevée. Il faut se souvenir que l'alter ego découvert dans l'empathie demeure bel et bien autre et que le « hic absolu » qu'il définit ne s'appréhende que dans une analogie. Le corps extérieur, même simplement représenté (celui du Christ, celui de Judas), parce qu'il est perçu dans une analogie avec mon propre corps, "acquiert par suite la signification d'un organisme se trouvant dans un autre "monde" analogue à mon monde primordial $»^{10}$. À suivre Husserl, il apparaît clairement que l'empathie ne permet en aucun cas l'assimilation de l'ici et du là. L'accès entier au hic absolu que constitue autrui reste barré :

Puisque le corps étranger (illic) entre dans un accouplement associatif avec mon corps (hic) et, donné dans la perception, devient le noyau d'une apprésentation - celle de l'expérience d'un ego co-existant - ce dernier doit nécessairement être apprésenté, conformément à tout le cours de l'association qui constitue son sens, comme un ego qui coexiste en ce moment dans le mode de l'illic ("comme si, moi, j'étais là-bas ») ${ }^{11}$.

L'empathie n'est pas la présence de l'ici dans le là, seulement l'apprésentation d'un autre ici absolu. Ce n'est donc ni l'aspiration totale par l'autre ni son envahissement par l'ego. La confusion de l'un et de l'autre, de l'ici et du là, n'est donc pas possible. Reconnaître l'autre, c'est au contraire admettre qu'il nous échappe - qu'il peut nous échapper - jusque dans le sentiment d'accéder à ce qu'il vit. Le « comme si » du texte husserlien s'avère donc fondamental.

C'est ce que la description de la fresque de Padoue permet encore d'illustrer : la résistance que nous pouvons ressentir, malgré l'empathie liée à «l'humanisme» de la peinture de Giotto, s'indique symboliquement dans le face à face entre le Christ et son apôtre. Si les deux corps semblent encore - dans l'écho de leur étreinte - ne faire qu'un, la séparation est nette et les quelques centimètres qui séparent objectivement leurs visages respectifs ne sont rien eu égard au gouffre absolu qui se creuse entre eux. De même donc que ces corps demeurent

\footnotetext{
${ }^{10}$ E. Husserl, Méditations cartésiennes, op. cit., p. 192.

11 Ibid., p. 193.
} 
séparés jusque dans leur proximité apparente, notre propre distance à l'égard des personnages de la fresque doit être réaffirmée, malgré l'empathie qui nous les donne dans une étrange proximité.

La conclusion de notre interrogation ne peut donc être qu'ambivalente. La représentation esthétique et l'empathie nous invitent à envisager une remise en question pour le moins étrange de l'absoluité de l'ici charnel. Ces expériences, dans leurs liens réciproques, nous font vivre, à un premier niveau, une paradoxale relativisation du centre que nous constituons: apparemment, ce centre se périphérise, devient flottant et indécis, tant parce que le là de l'œuvre nous aspire que parce qu'il nous semble pouvoir accéder au centre d'une autre sphère propre. Mais, à un second niveau d'analyse, il apparaît que ce flottement ou cette indécision est tout le contraire d'une relativisation de l'absolu.

La reconnaissance de la distance incompressible d'ici à là doit plutôt nous amener à réaffirmer la puissance charnelle à l'œuvre dans la constitution de l'espace. La force de l'ici absolu s'indique dans son pouvoir de décentrement, dans sa capacité à (quasi) sortir de soi : la non-coïncidence stricte de la chair et du corps rend possible une reconfiguration charnelle de l'espace dans laquelle l'ici absolu semble se donner par-delà le corps, jusque dans le corps de l'autre. Autrement dit, la puissance charnelle réside dans sa capacité à reconfigurer le corps, à le recentrer différemment (souvenons-nous de l'exemple du pied cassé), mais aussi à faire comme si elle pouvait se recentrer jusque dans le corps de l'autre.

Autrement dit encore, c'est dans le dynamisme incoercible de la reconfiguration charnelle que l'espace peut trouver quelque chose comme un absolu. La chair est ce qui, sans cesse, invente et réinvente le centre, par-delà l'ordre strict des corps, par-delà même les limites du corps auquel elle est nécessairement liée. De sorte que ce centre est moins une nécessité effective qu'une réinvention toujours possible.

À cet égard, la compréhension phénoménologique de la chair retrouve ce que l'anthropologie religieuse a par ailleurs mis en évidence : dans sa lecture de Tertullien, Serge Margel note ainsi l'importance, pour la compréhension chrétienne de la chair, de la notion d'adventus: "L'adventus tient donc en équilibre et opère une tension, propre au corps de chair justement, entre la présence et l'apparition ${ }^{12}$. Le parallèle se soutient ici, du moins dans la mesure où la chair se tient si loin de l'effectivité qu'elle n'est pas tant ce qui est que ce qui, toujours, advient. On pourrait dire: non pas tant le donné que le

\footnotetext{
12 S. Margel, L'invention du corps de chair, Étude d'anthropologie religieuse du premier christianisme, Paris, Éditions du Cerf, 2016, p. 66.
} 
donnant. Si elle se donne, ce n'est que dans une apparition, un surgissement étonnant qui reconfigure l'expérience.

Il nous semble que l'expérience esthétique et l'empathie constituent des épreuves remarquables de cette «advenue» de la chair : dans le décentrement/recentrement propre à ces expériences, la chair "dépasse les bornes » et s'arrache aux contours circonstanciés du corps objectif. L'assurance de présence effective du corps est alors comme balayée par l'absolu charnel.

Sentir l'espace se recentrer, notamment dans la contemplation esthétique et l'empathie, c'est donc raviver la chair, relativiser nos déterminations physiques, sentir le corps propre déborder le corps objectif, et ainsi, rendre à l'espace - et donc à l'expérience - la dimension de possible, par-delà l'effectivité. La "recentration » toujours possible de l'espace et l'impossibilité d'assimiler l'ici et le là signifient l'irréductibilité de l'expérience aux déterminations effectives, et l'impossibilité d'enfermer l'existence spatiale dans la présence physique des corps.

\section{L'espacement et la rencontre}

Cette réaffirmation de l'irréductibilité de l'ici au là renforce sans doute l'absoluité de la puissance charnelle, mais met peut-être en péril la possibilité de toute rencontre effective. Existe-t-il en effet un espace pour la rencontre? À défaut d'espace objectif, un véritable espace intersubjectif est-il phénoménologiquement possible? Ou l'espace se ramène-t-il à une séparation et un cloisonnement de l'ici et du là ? Sans doute, malgré la possibilité de l'empathie, les sphères propres ne peuvent-elles s'identifier, de sorte que l'espace phénoménologique demeure marqué par un espacement irréductible. Reste à se demander si cet espacement empêche véritablement la rencontre.

Sur ce point encore, la contemplation de la chapelle des Scrovegni peut nous être utile, afin de bien comprendre le statut de l'espacement qui s'avère au cœur de la compréhension phénoménologique de l'espace.

Comme on l'a dit, le visage du Christ et celui de Judas se touchent presque (de profil, leurs nez respectifs sont dans un quasi contact), mais cet écart, aussi infime soit-il, suggère, dans la dynamique d'éclatement et de rayonnement du tableau (cf. la position des lances et flambeaux), un tragique de la séparation, qui ne peut que se creuser. L'espacement correspond donc bien à une dynamique incoercible d'éloignement et un gouffre absolu déchire ici la proximité effective. 
L'espace charnel sépare ce qu'une certaine continuité et une certaine homogénéité physique rassemblent.

Si donc Giotto est de ces peintres qui inventent l'espace pictural nouveau de la Renaissance - espace qui, dans l'élaboration de la perspective, acquerra progressivement une certaine profondeur et une certaine homogénéité - il importe de bien comprendre que ce nouvel espace ne peut se réduire au continuum que l'on veut parfois y voir. Si la peinture de Giotto réalise une hypotypose si touchante, ce n'est pas tant parce qu'elle commence à construire cette profondeur (ici dans la succession et l'opposition colorée des plans) et cette homogénéité, que parce que, à l'intérieur même de ce continuum physique encore primitif, elle indique la possibilité de l'espacement charnel.

D'une certaine manière, il nous faut ici reprendre la thèse que Daniel Arasse ${ }^{13}$ exposait dans ses études sur la représentation picturale d'un autre thème, celui de l'Annonciation, pendant la Renaissance italienne. De même que l'espace perspectif de l'Annonciation italienne, malgré son homogénéité apparente, continue d'une certaine manière à poser des lieux distincts (en retirant notamment l'infini divin du régime de la visibilité), il nous semble que la quasi-homogénéité de l'espace pictural chez Giotto n'exclut en rien la représentation de la discontinuité phénoménologique des lieux charnels.

Sans doute même nous faut-il ici reconnaître un nouveau paradoxe : la représentation de l'espacement est cela même qui permet la rencontre. Si en effet nous pouvons regarder la fresque avec empathie, c'est parce que nous nous reconnaissons dans cet espacement. La figuration sensible de l'espacement de Judas et du Christ est cela même qui rend possible notre propre rencontre avec le tableau, dans la mesure où nous pouvons nous reconnaître dans cette expérience charnelle. Autrement dit : si cette peinture est mimétique, ce n'est pas tant parce qu'elle cherche à imiter les formes tridimensionnelles de la perception réelle que parce qu'elle donne à voir la dissociation de la chair et du corps, ainsi que la capacité de la chair à se recentrer et reconfigurer l'expérience.

Ajoutons que la rencontre qui se joue dans cet espacement est autant la découverte de l'ici que celle du là. Car ce n'est pas que nous projetons, là-bas, quelque chose que nous connaissions déjà ici : l'empathie que nous pouvons ressentir pour le Christ ou pour Judas ne nécessite ainsi pas que nous ayons déjà vécu une expérience identique à la leur. Ce que nous reconnaissons, c'est une possibilité de la différence charnelle que nous n'avons pas forcément réellement

${ }^{13}$ D. Arasse, L'annonciation italienne, Une histoire de perspective, Paris, Hazan, 2010. 
vécue. L'ici charnel fait donc une nouvelle fois preuve d'une puissance pour le moins étonnante : l'espacement d'avec le là de l'autre ne l'empêche pas de se reconnaître dans ce qu'elle ne connaît pas effectivement. L'espacement a beau suggérer un éloignement croissant d'ici à là, il n'empêche pas la rencontre comme assomption d'un possible pour l'ici charnel. En d'autres termes, l'espacement qui se fait jour malgré la proximité empathique n'élimine en rien la possibilité de la rencontre, en tant que celle-ci désigne essentiellement la confrontation avec la nouveauté d'une possibilité charnelle.

Ajoutons, pour finir, que l'espacement ne désigne pas seulement ce mouvement d'éloignement continu. L'espacement peut en effet se voir à l'œuvre, au contraire, dans la proximité paroxystique de l'étreinte.

Tournons-nous, pour nous en convaincre, vers une autre fresque de la chapelle des Scrovegni : celle qui représente le baiser échangé par Joachim et Anne lors de La rencontre à la Porte d'or. Si la représentation du baiser de Judas insiste sur la séparation, celle du baiser $\mathrm{d}^{\prime}$ Anne et Joachim met en scène l'union qui symbolise la naissance à venir de la Vierge : leurs auréoles respectives se fondent, leurs profils se joignent et leurs corps offrent une belle symétrie dans l'étreinte.

Mais cette union, il faut y insister, n'est pas une fusion. Regardons notamment la façon dont les deux visages réunis sont représentés. $\mathrm{D}^{\prime}$ une certaine manière, ces deux visages n'en font plus qu'un (l'œil droit de Joachim et l'œil gauche d'Anne composent un nouveau regard, comme les moitiés de l'une et l'autre bouche en dessinent une nouvelle). Mais ce nouveau visage n'en est pas un : il a quelque chose de monstrueux, comme si une sorte de tête cubiste au regard louche était là égarée dans la peinture de la Renaissance primitive. En outre, le cerne qui dessine le profil de Joachim insiste nettement sur la séparation dans l'union, de sorte que l'ici reste démarqué du là et que leur confusion n'est pas possible.

Enfin, l'union ici représentée est d'autant moins la fusion que l'on se tient peut-être à la limite (nous sommes à la «Porte» d'or) de la séparation de l'ici et du là. Le baiser unit mais maintient au seuil d'une fusion qui demeure impossible. Autrement dit, même si l'espacement ne tient plus qu'à un fil - il ne tient "qu'à un cerne » - il demeure inscrit dans la rencontre charnelle. L'espacement phénoménologique n'est donc décidément pas ce qui interdit la rencontre : car le baiser qui rassemble ici les époux séparés n'élimine pas tout-à-fait la séparation. 


\section{Conclusion : la séparation des a(i)mants}

Comment donc comprendre cet espacement d'ici à là et la possibilité de l'union malgré la séparation? Une comparaison nous semble de nature à saisir l'aspect dynamique de l'espacement phénoménologique : celle de la séparation des aimants. Deux aimants ont beau se repousser dans l'analogie de leur polarité, il est un écartement qui fait ressentir leur union dans la séparation. Leur espacement n'est alors plus un vide interstitiel, car il est éprouvé (par les mains qui maintiennent les aimants) comme un champ de tensions réciproques. De façon parallèle, le fait de retenir deux aimants qui s'attirent fait ressentir un certain écartement capable d'équilibrer l'attirance.

L'espace phénoménologique peut s'envisager de façon analogue à ces jeux de tensions, dans la mesure où l'espacement entre ici et là désigne une bipolarité qui semble aussi indépassable qu'elle est fragile et fondamentalement dynamique. À partir de la distinction de la chair et du corps, la phénoménologie peut donc décrire un espacement nécessaire qui demeure fondamentalement ambigu en raison de sa nature plurielle et dynamique. Ce faisant, elle nous rappelle au vécu d'une topologie charnelle de l'espace qui ne peut se réduire à son homogénéisation physique : la séparation des a(i)mants est décidément plus profonde que l'indifférence des corps assimilés.

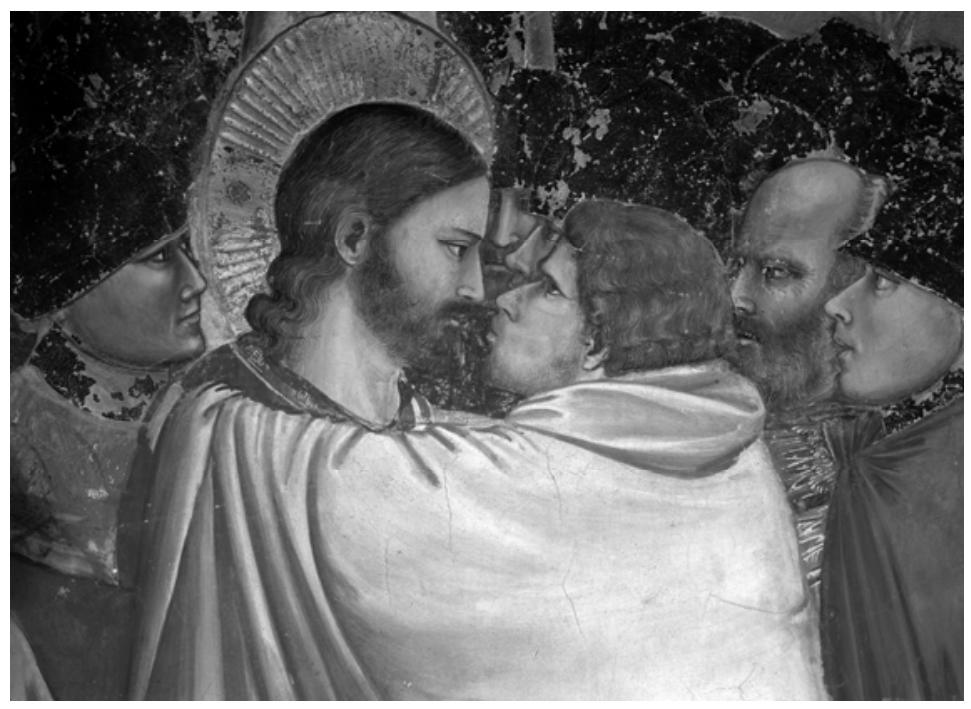

Giotto, Le Baiser de Judas (détail), 1303-1306, fresque, chapelle des Scrovegni, Padoue. 